\title{
Generalized Wideband Cyclic MUSIC
}

\author{
Zhang-Meng Liu, Zhi-Tao Huang, and Yi-Yu Zhou \\ Department of Electronic Science and Engineering, National University of Defense Technology of China, Changsha, \\ Hunan 410073, China \\ Correspondence should be addressed to Zhang-Meng Liu, liuzmcn@yahoo.cn
}

Received 7 March 2009; Revised 8 June 2009; Accepted 12 August 2009

Recommended by Irene $\mathrm{Gu}$

The method of Spectral Correlation-Signal Subspace Fitting (SC-SSF) fails to separate wideband cyclostationary signals with coherent second-order cyclic statistics (SOCS). Averaged Cyclic MUSIC (ACM) method made up for the drawback to some degree via temporally averaging the cyclic cross-correlation of the array output. This paper interprets ACM from another perspective and proposes a new DOA estimation method by generalizing ACM for wideband cyclostationary signals. The proposed method successfully makes up for the aforementioned drawback of SC-SSF and obtains a more satisfying performance than ACM. It is also demonstrated that ACM is a simplified form of the proposed method when only a single spectral frequency is exploited, and the integration of the frequencies within the signal bandwidth helps the new method to outperform ACM.

Copyright ( 2009 Zhang-Meng Liu et al. This is an open access article distributed under the Creative Commons Attribution License, which permits unrestricted use, distribution, and reproduction in any medium, provided the original work is properly cited.

\section{Introduction}

Most of the communication and radar signals show a special kind of periodicity called cyclostationarity because of artificial coding and modulation [1]. Gardner introduced cyclostationarity into array signal processing and proposed the method of Cyclic MUSIC and Cyclic ESPRIT [2]. Charge et al. extended Cyclic MUSIC by exploiting both the cyclic correlation and conjugate cyclic correlation of the array output [3]. Those methods combined the spatial information within the geometrically distributed array and temporal property of cyclostationarity within the incident signal waveform and thus become signal-selective to separate spatially adjacent signals with different cyclostationarity, but the method is limited only to narrowband signals. When the bandwidth of the signals increases, the estimates from Cyclic methods will become biased and modifications of the methods are required $[4,5]$.

After that, Xu and kailath concluded that a time delay in a signal only brings a corresponding phase delay to its cyclic autocorrelation, which is applicable to both narrowband and wideband cyclostationary signals [6]. Xu and kailath then used the cyclic autocorrelation of the array output to estimate the DOA of wideband signals; the proposed method is named Spectral Correlation-Signal Subspace Fitting (SC-SSF) [6]. SC-SSF is still signal-selective as Cyclic MUSIC and applies to both narrowband and wideband signals, but as it exploits only the cyclic autocorrelation of the array output, it fails to separate independent signals with the same modulation character $[7,8]$. Yan and Fan analyzed why such drawback occurs and introduced the idea of temporal averaging to make cyclic cross-correlation, which is previously used only for narrowband signals, applicable to wideband signals, and proposed the method of Averaged Cyclic MUSIC (ACM). The exploitation of the cyclic cross-correlation of the array output also makes ACM capable in separating signals with coherent SOCS $[7,8]$.

This paper interprets the method of ACM as a realization of Cyclic MUSIC for wideband signals at a certain spectral frequency. Further analysis shows that ACM can be generalized to an arbitrary spectral frequency within the signal bandwidth. But as the ACM method works at a single spectral frequency and exploits only part of the information contained in the incident signal waveform, its performance is suboptimal, and integration of the information within the signal bandwidth is expected to obtain an improved performance over that of ACM. 
This paper is organized as follows. Previous methods of SC-SSF and ACM are briefly reviewed in Section 2. Another interpretation of ACM and the principle of the generalized wideband Cyclic MUSIC method are detailed in Section 3. In Section 4 numerical examples are carried out to verify the effectiveness of the proposed method, and the whole paper is concluded in Section 5.

\section{Previous Wideband Cyclic Methods}

Suppose that a sensor array consisting of $M$ identical omnidirectional antennas is used to estimate the directions of the incident signals, and among the impinging signals, $K$ independent ones from the directions of $\theta_{1}, \ldots, \theta_{K}$ share the same cyclic frequency of $\alpha$, which is of interest, while the others are deemed as interferential ones that are cyclically independent to each other and to the signals at the cyclic frequency of $\alpha$. The methods discussed below apply to arbitrary array shapes, but in this paper only the uniform linear array (ULA) is considered for convenience.

2.1. SC-SSF Method [6]. Choose the first element of the array as the reference; the array output at the $m$ th element is

$$
x_{m}(t)=\sum_{k=1}^{K} s_{k}\left(t+(m-1) \Delta_{k}\right)+n_{m}(t),
$$

in which $s_{k}(t)$ is the signal waveform of the $k$ th source at time $t, \Delta_{k}=D \sin \theta_{k} / C$ is the time-delay of the $k$ th signal propagating between adjacent elements, $D$ is the interelement spacing, $C$ is the propagating velocity of the electromagnetic wave, and $n_{m}(t)$ is the mixture of the interferences and additive noise at the $m$ th channel.

The cyclic autocorrelation of the array output at the $m$ th element is

$$
\begin{aligned}
r_{x_{m} x_{m}}^{\alpha}(\tau) & =\left\langle x_{m}\left(t+\frac{\tau}{2}\right) x_{m}^{*}\left(t-\frac{\tau}{2}\right) e^{-j 2 \pi \alpha t}\right\rangle_{t} \\
& =\sum_{k=1}^{K} r_{s_{k} s_{k}}^{\alpha}(\tau) e^{j 2 \pi \alpha(m-1) \Delta_{k}},
\end{aligned}
$$

in which $\alpha$ is the cyclic frequency, $\tau$ is the time-delay, $\langle\bullet\rangle_{t}$ stands for temporal averaging, and $r_{s_{k} s_{k}}^{\alpha}(\tau)$ is the cyclic autocorrelation of the $k$ th signal at the cyclic frequency of $\alpha$ and time-delay of $\tau$, that is,

$$
r_{s_{k} s_{k}}^{\alpha}(\tau)=\left\langle s_{k}\left(t+\frac{\tau}{2}\right) s_{k}^{*}\left(t-\frac{\tau}{2}\right) e^{-j 2 \pi \alpha t}\right\rangle_{t}
$$

It can be concluded from (2) that as the interferences and noise are cyclically independent to each other and to the signals at the cyclic frequency $\alpha$, they cast no influence on the array output cyclic autocorrelation.
Then the cyclic autocorrelation of the whole array output at time-delay $\tau$ is given by

$$
\begin{aligned}
\mathbf{R}_{\mathbf{X X}}^{\alpha}(\tau)= & {\left[r_{x_{1} x_{1}}^{\alpha}(\tau), r_{x_{2} x_{2}}^{\alpha}(\tau), \ldots, r_{x_{M} x_{M}}^{\alpha}(\tau)\right]^{T} } \\
= & {\left[\sum_{k=1}^{K} r_{s_{k} s_{k}}^{\alpha}(\tau), \sum_{k=1}^{K} r_{s_{k} s_{k}}^{\alpha}(\tau) e^{j 2 \pi \alpha \Delta_{k}}, \ldots,\right.} \\
& \left.\sum_{k=1}^{K} r_{s_{k} s_{k}}^{\alpha}(\tau) e^{j 2 \pi \alpha(M-1) \Delta_{k}}\right]^{T} \\
= & \mathbf{A}(\alpha, \Theta) \mathbf{r}_{s}^{\alpha}(\tau),
\end{aligned}
$$

in which $\Theta=\left[\theta_{1}, \theta_{2}, \ldots, \theta_{K}\right]$ and

$$
\begin{gathered}
\mathbf{A}(\alpha, \Theta)=\left[\mathbf{a}\left(\alpha, \theta_{1}\right), \mathbf{a}\left(\alpha, \theta_{2}\right), \ldots, \mathbf{a}\left(\alpha, \theta_{K}\right)\right], \\
\mathbf{a}\left(\alpha, \theta_{k}\right)=\left[1, e^{j 2 \pi \alpha \Delta_{k}}, \ldots, e^{j 2 \pi \alpha(M-1) \Delta_{k}}\right]^{T}, \quad k=1, \ldots K
\end{gathered}
$$

is the array response to the incident signals, and

$$
\mathbf{r}_{\mathbf{s s}}^{\alpha}(\tau)=\left[r_{s_{1} s_{1}}^{\alpha}(\tau), r_{s_{2} s_{2}}^{\alpha}(\tau), \ldots, r_{s_{K} s_{K}}^{\alpha}(\tau)\right]^{T}
$$

is the vector of the cyclic autocorrelations of the incident signals.

When the autocorrelation of (4) is computed at different time-delays $\tau$ 's, a group of $\mathbf{R}_{\mathbf{X X}}^{\alpha}(\tau)$ can be obtained to form a pseudodata matrix as

$$
\begin{aligned}
\mathbf{Y}_{\mathrm{SC}-\mathrm{SSF}}(\alpha) & =\left[\mathbf{R}_{\mathbf{X X}}^{\alpha}(0), \mathbf{R}_{\mathbf{X X}}^{\alpha}\left(T_{s}\right), \ldots, \mathbf{R}_{\mathbf{X X}}^{\alpha}\left((L-1) T_{s}\right)\right] \\
& =\mathbf{A}(\alpha, \Theta)\left[\mathbf{r}_{\text {ss }}^{\alpha}(0), \mathbf{r}_{\mathbf{s s}}^{\alpha}\left(T_{s}\right), \ldots, \mathbf{r}_{\mathbf{s s}}^{\alpha}\left((L-1) T_{s}\right)\right] \\
& \triangleq \mathbf{A}(\alpha, \Theta) \mathbf{R}_{\mathbf{s s}}^{\alpha}(\mathbf{T}),
\end{aligned}
$$

in which $\mathbf{R}_{\mathbf{s s}}^{\alpha}(\mathbf{T})=\left[\mathbf{r}_{\mathrm{ss}}^{\alpha}(0), \mathbf{r}_{\mathbf{s s}}^{\alpha}\left(T_{s}\right), \ldots, \mathbf{r}_{\mathbf{s s}}^{\alpha}\left((L-1) T_{s}\right)\right]$ and $\mathbf{T}=$ $\left.\left\{l T_{s}\right\}\right|_{l=0, \ldots, L-1}$ is the set of time-delays.

Thus general narrowband subspace methods, such as MUSIC, can be applied to estimate the directions of the impinging wideband cyclostationary signal if $\mathbf{Y}_{\text {SC-SSF }}(\alpha) \mathbf{Y}_{\text {SC-SSF }}^{H}(\alpha)$ is of full rank.

There is a significant drawback in SC-SSF that if more than one incident signals share coherent SOCS, that is, for any time delay $\tau$, the linear dependence holds between the SOCS of two or more different signals as $r_{s_{i} s_{i}}^{\alpha}(\tau)=$ $\kappa r_{s_{j} s_{j}}^{\alpha}(\tau)(i \neq j)$ with $\kappa$ begin a constant, the $i$ th and $j$ th rows of $\mathbf{R}_{\mathrm{ss}}^{\alpha}(\mathbf{T})$ will be completely dependent; thus $\mathbf{R}_{\mathrm{ss}}^{\alpha}(\mathbf{T}) \mathbf{R}_{\mathrm{ss}}^{\alpha H}(\mathbf{T})$ and $\mathbf{Y}_{\mathrm{SC}-\mathrm{SSF}}(\alpha) \mathbf{Y}_{\mathrm{SC}-\mathrm{SSF}}^{H}(\alpha)$ become rank deficient and SC-SSF fails to resolve such signals. 
The drawback can be made up by substituting the cyclic cross-correlation for the autocorrelation to make the pseudodata matrix independent of the signal dependence, but it is hard to realize because the temporal envelope of the wideband signals is fast varying; thus the cross-correlation between different antenna outputs is channel-dependent and a DOA-dependent pseudodata matrix is no longer obtainable as the narrowband signals. This dissimilarity between wideband and narrowband signals can be concluded from the following inequality:

$$
\begin{aligned}
& r_{x_{p} x_{q}}^{\alpha}(\tau) \\
& =\left\langle x_{p}\left(t+\frac{\tau}{2}\right) x_{q}^{*}\left(t-\frac{\tau}{2}\right) e^{-j 2 \pi \alpha t}\right\rangle_{t} \\
& =\sum_{k=1}^{K}\left\langle a_{k}\left(t+\frac{\tau}{2}+(p-1) \Delta_{k}\right) a_{k}^{*}\left(t-\frac{\tau}{2}+(q-1) \Delta_{k}\right)\right. \\
& \left.\quad \times e^{j 2 \pi f_{0}\left(\tau+(p-q) \Delta_{k}\right)} e^{-j 2 \pi \alpha t}\right\rangle_{t} \\
& =\sum_{k=1}^{K} e^{j 2 \pi\left(f_{0}+\alpha / 2\right)(p-1) \Delta_{k}} r_{a_{k} a_{k}}^{\alpha}\left(\tau+(p-q) \Delta_{k}\right) \\
& \quad \times e^{-j 2 \pi\left(f_{0}-\alpha / 2\right)(q-1) \Delta_{k}} e^{j 2 \pi f_{0} \tau} \text { (wideband) } \\
& \neq \sum_{k=1}^{K} e^{j 2 \pi\left(f_{0}+\alpha / 2\right)(p-1) \Delta_{k}} r_{a_{k} a_{k}}^{\alpha}(\tau) \\
& \quad \times e^{-j 2 \pi\left(f_{0}-\alpha / 2\right)(q-1) \Delta_{k}} e^{j 2 \pi f_{0} \tau} \text { (narrowband), }
\end{aligned}
$$

in which $f_{0}$ is the carrier frequency and $a_{k}(t)(k=1, \ldots, K)$ are the amplitude envelope of the signals independent to each other, that is,

$$
s_{k}(t)=a_{k}(t) e^{j 2 \pi f_{0} t},
$$

and $a_{k}(t)$ are cyclically independent to each other as $s_{k}(t)$ for $k=1, \ldots, K$.

In the case of wideband signals, the cyclic correlation $r_{a_{k} a_{k}}^{\alpha}\left(\tau+(p-q) \Delta_{k}\right)$ for different row index $p$ and column index $q$ is distinct because of the fast varying signal envelope, and thus each element in the cyclic cross-correlation matrix of the array output is dependent on the corresponding row and column indices:

$$
\begin{aligned}
\mathbf{R}_{\mathbf{X X}}^{\alpha}(\tau)= & \left\{r_{x_{p} x_{q}}^{\alpha}(\tau)\right\}_{p, q=1, \ldots, M} \\
= & \left\{\sum_{k=1}^{K} e^{j 2 \pi\left(f_{0}+\alpha / 2\right)(p-1) \Delta_{k}} r_{a_{k} a_{k}}^{\alpha}\left(\tau+(p-q) \Delta_{k}\right)\right. \\
& \left.\times e^{-j 2 \pi\left(f_{0}-\alpha / 2\right)(q-1) \Delta_{k}} e^{j 2 \pi f_{0} \tau}\right\}_{p, q=1, \ldots, M}
\end{aligned}
$$

and no determinate relation in phase exists in the different rows of the matrix as that for narrowband signals shown in (11):

$$
\begin{aligned}
\mathbf{R}_{\mathbf{X X}}^{\alpha}(\tau)= & \left\{\sum_{k=1}^{K} e^{j 2 \pi\left(f_{0}+\alpha / 2\right)(p-1) \Delta_{k}} r_{a_{k} a_{k}}^{\alpha}(\tau)\right. \\
& \left.\times e^{-j 2 \pi\left(f_{0}-\alpha / 2\right)(q-1) \Delta_{k}} e^{j 2 \pi f_{0} \tau}\right\}_{p, q=1,2, \ldots, M} \\
= & \mathbf{A}\left(f_{0}+\frac{\alpha}{2}, \Theta\right) \operatorname{diag}\left\{r_{a_{k} a_{k}}^{\alpha}(\tau) e^{j 2 \pi f_{0} \tau}\right\}_{k=1, \ldots K} \\
& \times \mathbf{A}\left(f_{0}-\frac{\alpha}{2}, \Theta\right) .
\end{aligned}
$$

Thus the information about the DOA cannot be extracted directly from the cross-correlation matrix of the wideband signals in (10).

2.2. ACM Method [7]. In order to make up for the drawback in SC-SSF of failing to resolve signals with coherent SOCS, Yan introduced the idea of temporal averaging into the acquirement process of the cyclic cross-correlation of the array output for wideband signals and proposed the method of ACM. Such averaging works as follows:

$$
\begin{aligned}
& r_{x_{p} x_{q}}\left(\alpha, f_{0}\right) \\
& \triangleq\left\langle r_{x_{p} x_{q}}^{\alpha}(\tau) e^{-j 2 \pi f_{0} \tau}\right\rangle_{\tau} \\
& =\sum_{k=1}^{K} e^{j 2 \pi\left(f_{0}+\alpha / 2\right)(p-1) \Delta_{k}} \\
& \times\left\langle r_{a_{k} a_{k}}^{\alpha}\left(\tau+(p-q) \Delta_{k}\right) e^{j 2 \pi f_{0} \tau} e^{-j 2 \pi f_{0} \tau}\right\rangle_{\tau} \\
& \times e^{-j 2 \pi\left(f_{0}-\alpha / 2\right)(q-1) \Delta_{k}} \\
& =\sum_{k=1}^{K} e^{j 2 \pi\left(f_{0}+\alpha / 2\right)(p-1) \Delta_{k}} \hat{r}_{a_{k} a_{k}}\left(\alpha, f_{0}\right) e^{-j 2 \pi\left(f_{0}-\alpha / 2\right)(q-1) \Delta_{k}} \\
& =\left[e^{j 2 \pi\left(f_{0}+\alpha / 2\right)(p-1) \Delta_{1}}, \ldots, e^{j 2 \pi\left(f_{0}+\alpha / 2\right)(p-1) \Delta_{K}}\right] \\
& \times\left[\begin{array}{ccc}
\hat{r}_{a_{1} a_{1}}\left(\alpha, f_{0}\right) & & \\
& \ddots & \\
& & \hat{r}_{a_{K} a_{K}}\left(\alpha, f_{0}\right)
\end{array}\right]\left[\begin{array}{c}
e^{-j 2 \pi\left(f_{0}-\alpha / 2\right)(q-1) \Delta_{1}} \\
\vdots \\
e^{-j 2 \pi\left(f_{0}-\alpha / 2\right)(q-1) \Delta_{K}}
\end{array}\right],
\end{aligned}
$$

in which the averaging window is set to contain most of the energy within the cyclic correlation and the denotation $\hat{r}_{a_{i} a_{i}}\left(\alpha, f_{0}\right)$ is used to indicate that the variable is dependent only on $\alpha$ and $f_{0}$ and does not change with $\tau$. 
The averaged cyclic cross correlation of the array output is then given by

$$
\begin{aligned}
& \mathbf{Y}_{\mathrm{ACM}}\left(\alpha, f_{0}\right) \\
& \triangleq\left\langle\mathbf{R}_{\mathbf{X X}}^{\alpha}(\tau) e^{-j 2 \pi f_{0} \tau}\right\rangle_{\tau} \\
& =\left.\left\{r_{x_{p} x_{q}}\left(\alpha, f_{0}\right)\right\}\right|_{p, q=1, \ldots, M} \\
& =\left.\left\{\left\langle r_{x_{p} x_{q}}^{\alpha}(\tau) e^{-j 2 \pi f_{0} \tau}\right\rangle_{\tau}\right\}\right|_{p, q=1, \ldots, M} \\
& =\left[\begin{array}{ccc}
1 & \cdots & 1 \\
\vdots & \ddots & \vdots \\
e^{j 2 \pi\left(f_{0}+\alpha / 2\right)(M-1) \Delta_{1}} & \cdots & e^{j 2 \pi\left(f_{0}+\alpha / 2\right)(M-1) \Delta_{K}}
\end{array}\right] \\
& \times\left[\begin{array}{lll}
\hat{r}_{a_{1} a_{1}}\left(\alpha, f_{0}\right) & & \\
& \ddots & \\
& & \hat{r}_{a_{K} a_{K}}\left(\alpha, f_{0}\right)
\end{array}\right] \\
& \times\left[\begin{array}{ccc}
1 & \cdots & 1 \\
\vdots & \ddots & \vdots \\
e^{j 2 \pi\left(f_{0}-\alpha / 2\right)(M-1) \Delta_{1}} & \cdots & e^{j 2 \pi\left(f_{0}-\alpha / 2\right)(M-1) \Delta_{K}}
\end{array}\right]^{H},
\end{aligned}
$$

in which $\left.\{\bullet\}\right|_{p, q=1, \ldots, M}$ is the reshape operator to form an $M \times M$ matrix with $p$, the index of row, and $q$, the index of column.

It should be noted that Yan and Fan missed $e^{j 2 \pi f_{0} \tau}$ in $r_{x_{p} x_{q}}^{\alpha}(\tau)$ and obtained the same conclusion as (12) and (13) via direct temporal averaging [7] instead of appending a weight of $e^{-j 2 \pi f_{0} \tau}$.

The cyclic cross-correlation of the array output using temporal averaging can then be written in the form as

$$
\mathbf{Y}_{\mathrm{ACM}}\left(\alpha, f_{0}\right)=\mathbf{A}\left(f_{0}+\frac{\alpha}{2}, \Theta\right) \hat{\boldsymbol{\Lambda}}_{\mathbf{a a}}(\alpha) \mathbf{A}^{H}\left(f_{0}-\frac{\alpha}{2}, \Theta\right)
$$

in which $\mathbf{A}\left(f_{0}+\alpha / 2, \Theta\right)$ and $\mathbf{A}\left(f_{0}-\alpha / 2, \Theta\right)$ are defined similar to $\mathbf{A}(\alpha, \Theta)$ in (4) and the referential frequency is set accordingly, and $\hat{\Lambda}_{\mathrm{aa}}(\alpha)=\operatorname{diag}\left\{\hat{r}_{a_{1} a_{1}}(\alpha), \ldots, \hat{r}_{a_{K} a_{K}}(\alpha)\right\}$ is a diagonal matrix that depends on each signal envelope.

As the sampling is limited in practical applications, the averaging process is somewhat inaccurate, but the numerical examples showed that ACM proves outstanding in performance when appropriate time delays are taken for averaging [7].

\section{Generalized Wideband Cyclic MUSIC}

3.1. Another Interpretation of ACM. In ACM, temporal averaging operates on the cyclic cross-correlation of the array output with a weight of $e^{-j 2 \pi f_{0} \tau}$, which equals (15) from the perspective of time-frequency transformation:

$$
r_{x_{p} x_{q}}\left(\alpha, f_{0}\right)=\left\langle r_{x_{p} x_{q}}^{\alpha}(\tau) e^{-j 2 \pi f_{0} \tau}\right\rangle_{\tau}=S_{x_{p} x_{q}}^{\alpha}\left(f_{0}\right)
$$

in which $S_{x_{p} x_{q}}^{\alpha}\left(f_{0}\right)$ is the cyclic spectral correlation of $x_{p}$ and $x_{q}$ at cyclic frequency $\alpha$ and spectral frequency $f_{0}$.

And the averaged pseudodata matrix can be interpreted as in (16) accordingly:

$$
\mathbf{Y}_{\mathrm{ACM}}\left(\alpha, f_{0}\right) \triangleq\left\langle\mathbf{R}_{\mathbf{X X}}^{\alpha}(\tau) e^{-j 2 \pi f_{0} \tau}\right\rangle_{\tau}=\mathbf{S}_{\mathbf{X X}}^{\alpha}\left(f_{0}\right)
$$

Equation (16) indicates that ACM can be deemed as a direction finding method exploiting the signal information at cyclic frequency $\alpha$ and spectral frequency $f_{0}$.

3.2. Generalized Wideband Cyclic MUSIC. In (15) $r_{x_{p} x_{q}}\left(\alpha, f_{0}\right)$ is interpreted as the Fourier transformation of the temporal sequence $r_{x_{p} x_{q}}^{\alpha}(\tau)$ at a certain spectral frequency of $f_{0}$. In fact, this time-frequency can be generalized to an arbitrary frequency of $f$ within the signal bandwidth:

$$
\begin{aligned}
& r_{x_{p} x_{q}}(\alpha, f) \\
& =S_{x_{p} x_{q}}^{\alpha}(f)=\left\langle r_{x_{p} x_{q}}^{\alpha}(\tau) e^{-j 2 \pi f \tau}\right\rangle_{\tau} \\
& =\sum_{k=1}^{K} e^{j 2 \pi\left(f_{0}+\alpha / 2\right)(p-1) \Delta_{k}} \\
& \times\left\langle r_{a_{k} a_{k}}^{\alpha}\left(\tau+(p-q) \Delta_{k}\right) e^{j 2 \pi f_{0} \tau} e^{-j 2 \pi f \tau}\right\rangle_{\tau} \\
& \times e^{-j 2 \pi\left(f_{0}-\alpha / 2\right)(q-1) \Delta_{k}} \\
& =\sum_{k=1}^{K} e^{j 2 \pi\left(f_{0}+\alpha / 2\right)(p-1) \Delta_{k}}\left[s_{a_{k} a_{k}}^{\alpha}\left(f-f_{0}\right) e^{j 2 \pi\left(f-f_{0}\right)(p-q) \Delta_{k}}\right] \\
& \times e^{-j 2 \pi\left(f_{0}-\alpha / 2\right)(q-1) \Delta_{k}} \\
& =\sum_{k=1}^{K} e^{j 2 \pi(f+\alpha / 2)(p-1) \Delta_{k}} s_{a_{k} a_{k}}^{\alpha}\left(f-f_{0}\right) e^{-j 2 \pi(f-\alpha / 2)(q-1) \Delta_{k}} \\
& =\left[e^{j 2 \pi(f+\alpha / 2)(p-1) \Delta_{1}}, \ldots, e^{j 2 \pi(f+\alpha / 2)(p-1) \Delta_{K}}\right] \\
& \times\left[\begin{array}{lll}
s_{a_{1} a_{1}}^{\alpha}\left(f-f_{0}\right) & & \\
& \ddots & \\
& & s_{a_{K} a_{K}}^{\alpha}\left(f-f_{0}\right)
\end{array}\right] \\
& \times\left[\begin{array}{c}
e^{-j 2 \pi(f-\alpha / 2)(q-1) \Delta_{1}} \\
\vdots \\
e^{-j 2 \pi(f-\alpha / 2)(q-1) \Delta_{K}}
\end{array}\right] .
\end{aligned}
$$


And the averaged pseudodata matrix weighted by $e^{-j 2 \pi f \tau}$ is

$$
\begin{aligned}
& \mathbf{Y}_{\mathrm{ACM}}(\alpha, f) \\
& =\mathbf{S}_{\mathbf{X X}}^{\alpha}(f)=\left\langle\mathbf{R}_{\mathbf{X X}}^{\alpha}(\tau) e^{-j 2 \pi f \tau}\right\rangle_{\tau} \\
& =\left.\left\{r_{x_{p} x_{q}}(\alpha, f)\right\}\right|_{p, q=1, \ldots, M}=\left.\left\{\left\langle r_{x_{p} x_{q}}^{\alpha}(\tau) e^{-j 2 \pi f \tau}\right\rangle_{\tau}\right\}\right|_{p, q=1, \ldots, M} \\
& =\left[\begin{array}{ccc}
1 & \cdots & 1 \\
\vdots & \ddots & \vdots \\
e^{j 2 \pi(f+\alpha / 2)(M-1) \Delta_{1}} & \cdots & e^{j 2 \pi(f+\alpha / 2)(M-1) \Delta_{K}}
\end{array}\right] \\
& \times\left[\begin{array}{lll}
s_{a_{1} a_{1}}^{\alpha}\left(f-f_{0}\right) & & \\
& \ddots & \\
& & s_{a_{K} a_{K}}^{\alpha}\left(f-f_{0}\right)
\end{array}\right] \\
& \times\left[\begin{array}{ccc}
1 & \cdots & 1 \\
\vdots & \ddots & \vdots \\
e^{j 2 \pi(f-\alpha / 2)(M-1) \Delta_{1}} & \cdots & e^{j 2 \pi(f-\alpha / 2)(M-1) \Delta_{K}}
\end{array}\right]^{H} \\
& =\mathbf{A}\left(f+\frac{\alpha}{2}, \Theta\right) \mathbf{S}_{\mathbf{a a}}\left(\alpha, f-f_{0}\right) \mathbf{A}^{H}\left(f-\frac{\alpha}{2}, \Theta\right),
\end{aligned}
$$

in which $\mathbf{S}_{\mathrm{aa}}\left(\alpha, f-f_{0}\right)=\operatorname{diag}\left\{s_{a_{1} a_{1}}^{\alpha}\left(f-f_{0}\right), \ldots, s_{a_{K} a_{K}}^{\alpha}\left(f-f_{0}\right)\right\}$.

It is obvious that the method of ACM is a special form of the model given above at the spectral frequency of $f=$ $f_{0}$. As the spectrum of most wideband signals, such as the phase-shift-keying (PSK) ones, owns the most significant energy density at the center of their baseband spectrum or the carrier frequency of their modulated spectrum (i.e., $\left|s_{a_{k} a_{k}}^{\alpha}\left(f-f_{0}\right)\right|(k=1, \ldots, K)$ take their maximums at the cyclic frequency of the baud rate and spectral frequency of $f=f_{0}$ ), ACM obtains a satisfying performance even though only a single spectral frequency is exploited.

But as the wideband signals occupy a considerable spectral bandwidth, and different frequencies contain complementary information about the signal, there is inevitably a loss of information in the method of ACM which exploits only a certain spectral frequency, and an integrated consideration of the signal bandwidth is expected to gain improved performance.

Assume that $I$ discrete spectral frequencies $f_{1}, f_{2}, \ldots, f_{I}$ within the signal bandwidth are exploited to obtain independent pseudodata matrices, that is,

$$
\begin{array}{r}
\mathbf{Y}_{\mathrm{ACM}}\left(\alpha, f_{i}\right)=\mathbf{A}\left(f_{i}+\frac{\alpha}{2}, \Theta\right) \mathbf{S}_{\mathrm{aa}}\left(\alpha, f_{i}-f_{0}\right) \mathbf{A}^{H}\left(f_{i}-\frac{\alpha}{2}, \Theta\right) \\
i=1,2, \ldots, I .
\end{array}
$$

Then by eigen-decomposing $\mathbf{Y}_{\mathrm{ACM}}\left(\alpha, f_{i}\right) \mathbf{Y}_{\mathrm{ACM}}^{H}\left(\alpha, f_{i}\right)$ for $i=$ $1,2, \ldots, I$ separately, we can obtain $K$ eigen-vectors corresponding to the $K$ largest eigen-values that span a subspace identical to the column subspace of $\mathbf{A}\left(f_{i}+\alpha / 2, \Theta\right)$ at each frequency:

$$
\mathbf{Y}_{\mathrm{ACM}}\left(\alpha, f_{i}\right) \mathbf{Y}_{\mathrm{ACM}}^{H}\left(\alpha, f_{i}\right)=\left[\begin{array}{ll}
\mathbf{U}_{s}^{i} & \mathbf{U}_{n}^{i}
\end{array}\right]\left[\begin{array}{cc}
\sum_{s}^{i} & \\
& \sum_{n}^{i}
\end{array}\right]\left[\begin{array}{ll}
\mathbf{U}_{s}^{i} & \mathbf{U}_{n}^{i}
\end{array}\right]^{H},
$$

in which $\mathbf{U}_{s}^{i}$ is the $M \times K$ signal subspace spanning a subspace identical to the column space of the array responding matrix $\mathbf{A}\left(f_{i}+\alpha / 2, \Theta\right), \hat{\mathbf{U}}_{n}^{i}$ is the $M \times(M-K)$ noise subspace orthogonal to the signal subspace, and the eigen-vectors are arranged in the diagonal matrix of $\left[\begin{array}{ll}\sum_{s} & \\ & \sum_{n}\end{array}\right]$ in the descending order.

Then the directions of the incident signals can be estimated by evaluating the orthogonality between the noise subspace and the direction to be checked at each spectral frequency separately:

$$
\widehat{\Theta}_{\mathrm{i}}=\arg \min _{\Theta}\left|\hat{\mathbf{U}}_{n}^{i}{ }^{H} \mathbf{a}\left(f_{i}+\frac{\alpha}{2}, \theta\right)\right|_{2}^{2} .
$$

As a set of DOA estimates can be obtained separately at each spectral frequency, further integration of them is required to gain improved performance.

For conventional wideband signals, the methods such as ISSM [9] were used to integrate the information contained in signal components of different frequencies to improve the accuracy of the DOA estimates. Similarly, when the cyclic frequency is fixed, the cyclostationary signals occupy a wide bandwidth alike to that of conventional wideband signals; thus the frequencies fusing idea of ISSM can be extended to wideband cyclostationary signals, and the estimates are given by

$$
\widehat{\Theta}=\arg \min _{\Theta} \sum_{i=1}^{I} \mathbf{a}^{H}\left(f_{i}+\frac{\alpha}{2}, \theta\right) \hat{\mathbf{U}}_{N}^{i} \hat{\mathbf{U}}_{N}^{i}{ }^{H} \mathbf{a}\left(f_{i}+\frac{\alpha}{2}, \theta\right) .
$$

Thus the direction finding for wideband cyclostationary signals is realized. As this method can be deemed as an extension of that proposed by Yan and Fan with the name of wideband Cyclic MUSIC [7, 8], the proposed method is named generalized wideband Cyclic MUSIC hereafter.

It can be easily concluded from (22) that an improved performance of direction finding can be expected from the generalized wideband cyclic MUSIC method as more than one discrete frequencies are exploited, and the more effective frequencies exploited, the more the performance is improved. But during the realization of the proposed method, we have to recompute the temporally averaged cyclic cross-correlation of the array output given by (18) $I$ times to obtain the pseudodata matrix $\mathbf{Y}_{\mathrm{ACM}}\left(\alpha, f_{i}\right)$ at the $I$ spectral frequencies $f_{i}$ for $i=1,2, \ldots, I$, and then eigen-decompose its covariance to get the $\hat{\mathbf{U}}_{n}^{i} s$; so the more frequencies we use in the proposed method, the more 


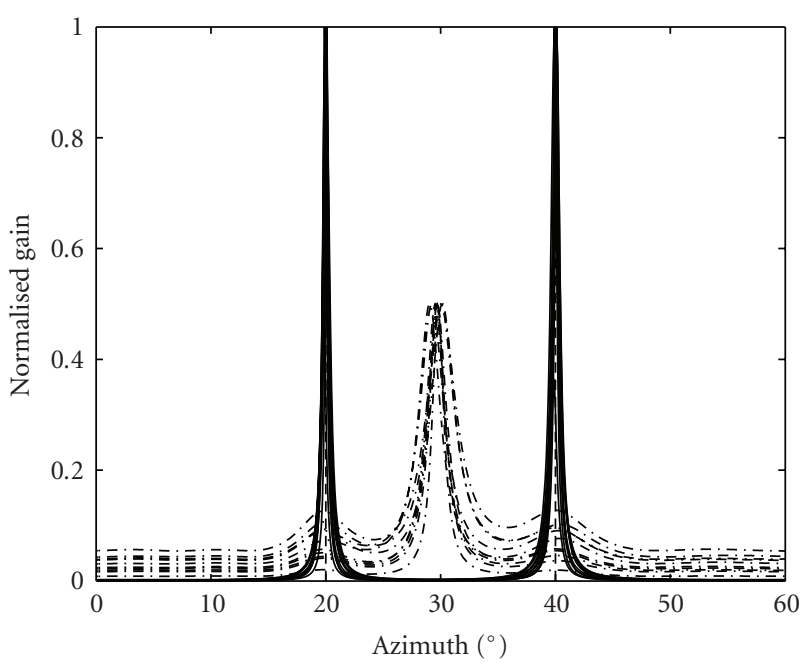

FIGURE 1: Spatial spectrums of the new method and SC-SSF for two impinging signals with coherent SOCS: -.- for SC-SSF; - for the proposed method.

expensive the computational burden will be. Therefore, when using the proposed method, there is a trade-off between the computational burden and the performance, and the specific frequencies to be chosen depend on both the computing speed of the practical system and the DOA estimating precision required. In the numerical experiments that follow, we choose several typical frequencies to verify the predominance of the proposed method over ACM.

\section{Numerical Examples}

In this section, three groups of numerical examples are carried out to show the satisfying performance of the proposed method, and much attention is paid to the dominance of the proposed method over SC-SSF and ACM when more than one incident signal that shares coherent SOCS impinges onto the array instantaneously. In the experiments, an 8-element ULA with an inter-element spacing of half the central wavelength is used; the impinging signals are independent BPSK ones with carrier frequency of $20 \mathrm{MHz}$ and their SOCS are coherent; the rates of sampling and width of sampling window are $80 \mathrm{MHz}$ and 0.1 milliseconds, respectively, that is, 8000 samples are used; autocorrelation of the array output with time-delays within \pm 30 sampling periods is considered in SC-SSF and taken for averaging in ACM and the proposed method, and in all of the three methods the cyclostationarity of the signals at the cyclic frequency of the baud rate is exploited.

4.1. Capability of Separating Signals with Coherent SOCS. In this experiment, two signals with the same SNR of $0 \mathrm{~dB}$ impinge onto the array from the directions of $20^{\circ}$ and $40^{\circ}$, respectively, the baud rate of them are both $10 \mathrm{MHz}$. For the proposed method, only the spectral frequency of $f=$ $f_{0}$ is used. Figure 1 shows the spatial spectrums within the azimuth scope from $0^{\circ}$ to $60^{\circ}$ in 10 independent trials of both the proposed method and SC-SSF.

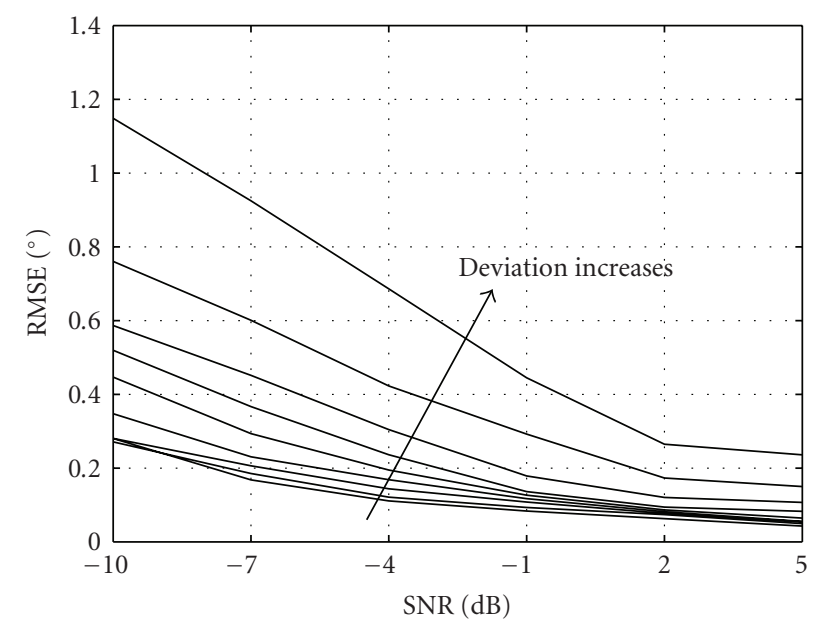

FIGURE 2: RMSE of the proposed method exploiting different single spectral frequencies.

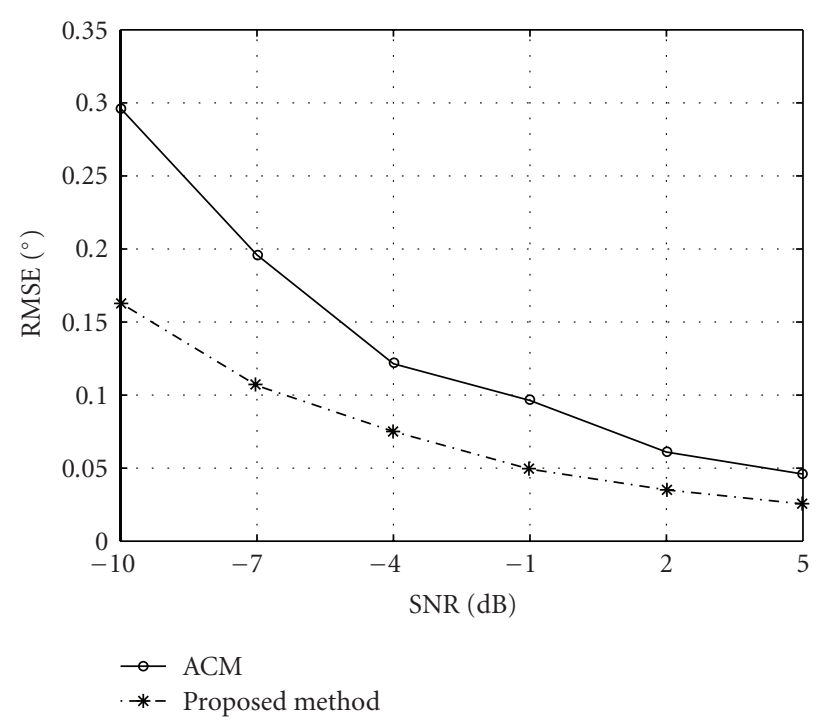

FIGURE 3: RMSE of the proposed method and ACM when one signal impinges onto the array.

In Figure 1 the spatial spectrum graphs in dot-dashed line correspond to SC-SSF and the ones in real line correspond to the proposed method. In the figure the proposed method separated the signals with coherent SOCS successfully while the method of SC-SSF fails to do so, which demonstrates that the proposed method is more robust than SC-SSF in such demanding signal circumstances.

4.2. Performance of the New Method Based on More Than One Spectral Frequency. We explained in Section 3 that the method of ACM outperforms other single spectral frequency-based methods because ACM exploits the signal component at the spectral frequency with the greatest energy density; this conclusion can be verified by the following experiment. 


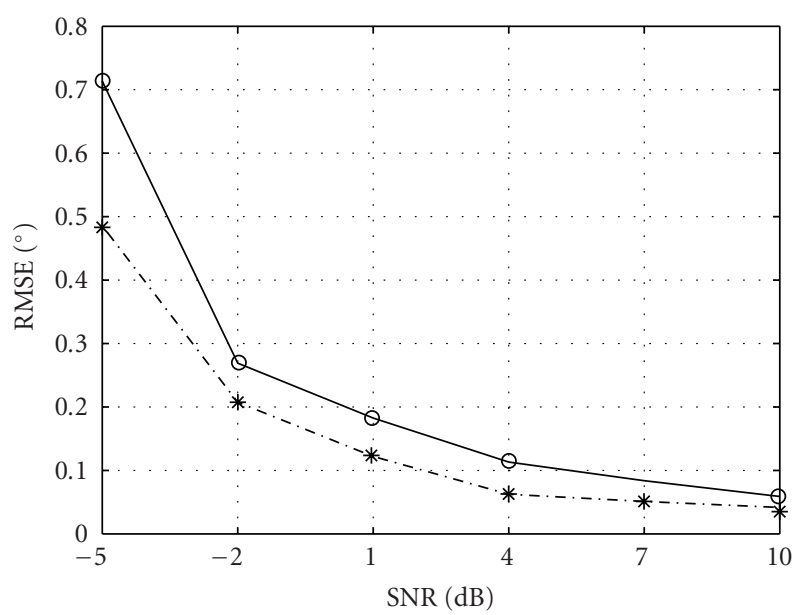

(a) $-3^{\circ}$ signal

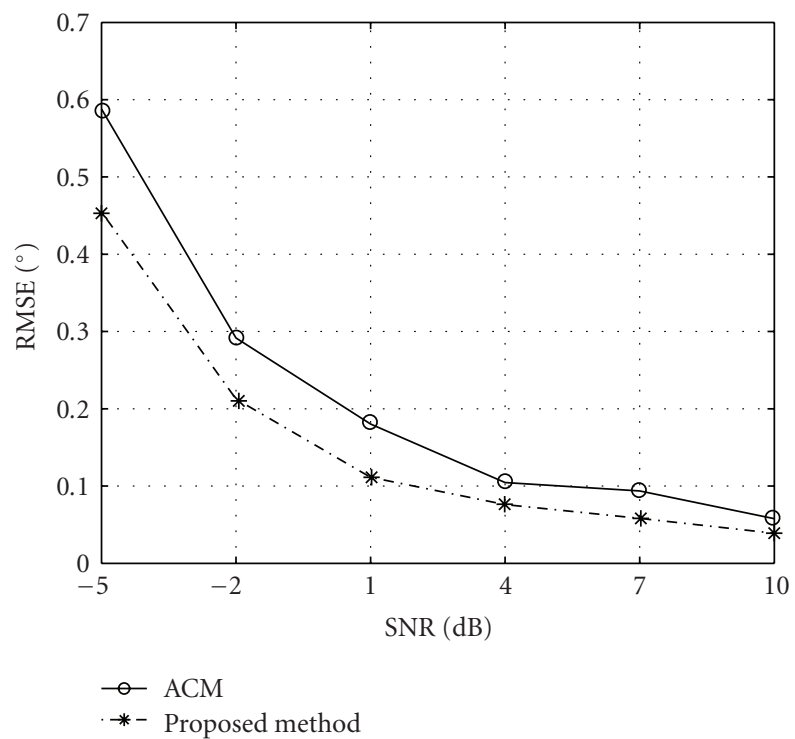

(b) $3^{\circ}$ signal

FIGURE 4: RMSE of the proposed method and ACM when two BPSK signals with baud rate of $10 \mathrm{MHz}$ impinge onto the array.

In this experiment, a BPSK signal with baud rate of $10 \mathrm{MHz}$ impinges from the direction of $0^{\circ}$ onto the array; the spectral frequencies for the weighted averaging of $\mathbf{R}_{\mathbf{X X}}^{\alpha}(\tau)$, that is, $f$ in (18), center at the carrier frequency and deviate from it by multiples of the baud rate from 0 to 0.4 with an interval of 0.05 . The performance of the method exploiting different single spectral frequencies is indicated by their rootmean-square-error (RMSE) obtained from 100 independent trials shown in Figure 2.

It is obvious in Figure 2 that the performance of the proposed method exploiting single spectral frequency deteriorates as the frequency deviates from the energy center of the signal bandwidth.

4.3. Dominance over ACM in Performance. The following three experiments verify the dominance of the proposed

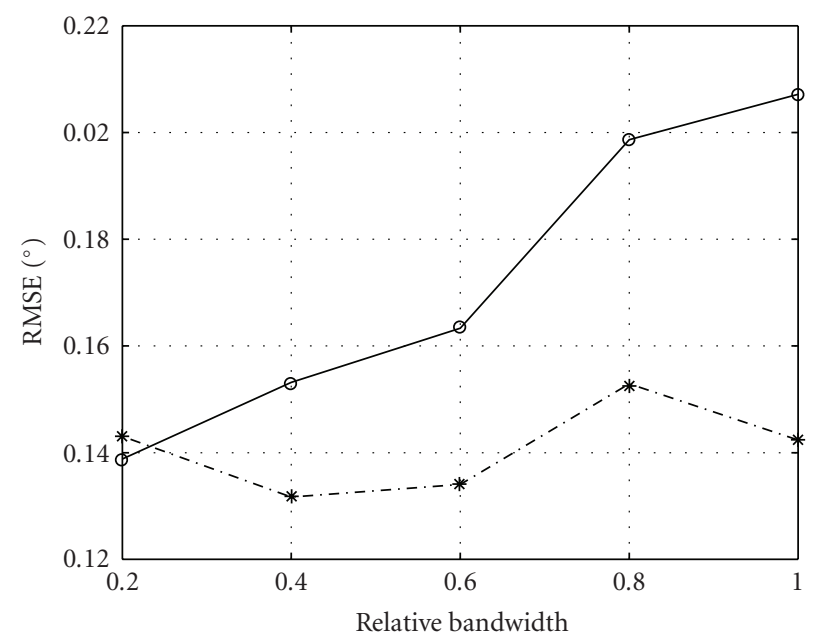

(a) $-3^{\circ}$ signal

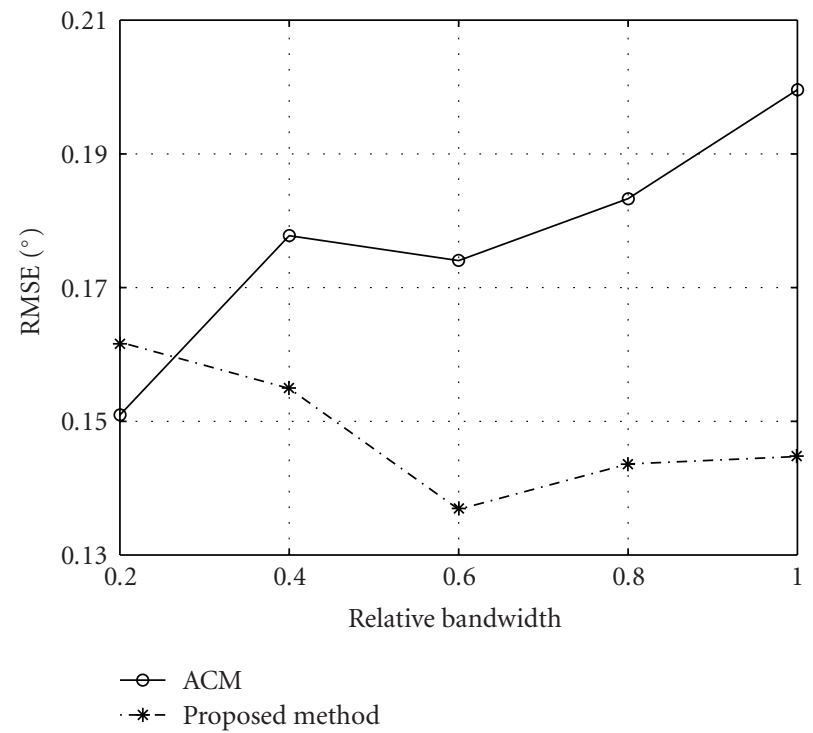

(b) $3^{\circ}$ signal

FIGURE 5: RMSE of the proposed method and ACM when two BPSK signals with SNR of $0 \mathrm{~dB}$ impinge onto the array.

method over ACM in performance when more than one spectral frequency is exploited. For both two methods the cross-correlation with time-delay within \pm 30 sampling periods is taken for averaging, and for the new method the spectral frequency of $f=f_{0}$ and four others centered at that point with deviations of \pm 0.1 and \pm 0.2 multiples of the baud rate is taken into account. The dash-dotted lines correspond to the performance of the new method while the real lines to ACM; the statistical performances are obtained from 100 independent trials.

Figure 3 gives the statistical result of the RMSE of one BPSK signal with baud rate of $10 \mathrm{MHz}$ impinging onto the array from the direction of $0^{\circ}$ and the SNR varies from $-10 \mathrm{~dB}$ to $5 \mathrm{~dB}$.

When two BPSK signals with the same baud rate of $10 \mathrm{MHz}$ impinge onto the array from $-3^{\circ}$ and $3^{\circ}$, respectively, and the SNR varies from $-5 \mathrm{~dB}$ to $10 \mathrm{~dB}$, Figure 4 
gives the RMSE of both signals obtained from the proposed method and ACM.

If the SNR is fixed at $0 \mathrm{~dB}$, the baud rates of the two signals vary from $2 \mathrm{MHz}$ to $10 \mathrm{MHz}$ and the cyclic frequency chosen varies accordingly; Figure 5 shows the performances of both the proposed method and ACM.

It can be concluded from Figures $3-5$ that the proposed method proves predominance over ACM in most cases as more than one spectral frequency is exploited because of effective exploitation of the information within the signal bandwidth. But if the bandwidth of the signals is not wide enough, the integration of different spectral frequencies will not achieve improvement in performance over ACM as is shown in Figure 5 when the relative signal bandwidth is small. The reason of this drawback is that the spectral energy density of those signals falls sharply as the frequency deviates from the center, and thus the information contained in the other signal spectral components may not complement much to the central frequency.

\section{Conclusions}

In this paper, the method of ACM is interpreted from another perspective and the reason why it works well is given, then it is extended to a general form that works on an arbitrary spectral frequency within the signal bandwidth at a certain cyclic frequency, and a way of integrating the information contained at different spectral frequencies is given to obtain a new method named generalized wideband Cyclic MUSIC with an improved performance over ACM. Simulation results show that the proposed method successfully makes up for the drawback of SC-SSF in failing to resolve signals with coherent SOCS, and integration of different spectral frequencies helps the proposed method to surpass ACM in performance.

\section{Acknowledgments}

This work is supported by the National Natural Science Foundation of China (no. 60502040), Hunan Provincial Innovation Foundation for Postgraduates, and the Innovation Foundation for Outstanding Postgraduates in the National University of Defense Technology of China.

\section{References}

[1] W. A. Gardner, Cyclostationarity in Communications and Signal Processing, IEEE Press, New York, NY, USA, 1994.

[2] W. A. Gardner, "Simplification of MUSIC and ESPRIT by exploitation of cyclostationarity," Proceedings of the IEEE, vol. 76, no. 7, pp. 845-847, 1988.

[3] P. Charge, Y. Wang, and J. Saillard, "An extended cyclic MUSIC algorithm," IEEE Transactions on Signal Processing, vol. 51, no. 7, pp. 1695-1701, 2003.

[4] H. Yan and H. H. Fan, "Improved cyclic and conjugate cyclic MUSIC," in Processings of the IEEE Sensor Array and Multichannel Signal Processing Workshop, pp. 289-293, July 2004.
[5] H. Yan and H. H. Fan, "On improvements of cyclic MUSIC," EURASIP Journal on Applied Signal Processing, vol. 2005, no. 1, pp. 61-68, 2005.

[6] G. H. Xu and T. Kailath, "Direction-of-arrival estimation via exploitation of cyclostationary-a combination of temporal and spatial processing," IEEE Transactions on Signal Processing, vol. 40, no. 7, pp. 1775-1786, 1992.

[7] H. Yan and H. H. Fan, "Wideband cyclic MUSIC algorithms," Signal Processing, vol. 85, no. 3, pp. 643-649, 2005.

[8] H. Yan and H. H. Fan, "Extended wideband cyclic MUSIC," in Processings of the IEEE Sensor Array and Multichannel Signal Processing Workshop, pp. 318-322, July 2004.

[9] M. Wax and T. Kailath, "Spatio-temporal spectral analysis by eigenstructure methods," IEEE Transactions on Acoustics, Speech, and Signal Processing, vol. 32, no. 4, pp. 817-827, 1984. 